\title{
CRISIS EN LA PAREJA
}

\author{
Ana Escobedo Ríos, Mg. \\ Psicóloga clínica - Terapeuta familiar \\ Universidad Peruana Unión \\ escorry@hotmail.com
}

Fecha de recepción: Mayo 2012 Fecha de aceptación y versión final: Julio 2012

Resumen $\mid$ Los matrimonios enfrentan crisis de diferentes tipos. El presente artículo divide las crisis de par eja en crisis vitales y crisis inesper adas. A partir de esa división, se hace un recorrido por las diferentes causas de crisis en el matrimonio, y se pr opone algunos consejos $v$ aliosos que ayudarán a los casados a fortalecer su matrimonio usando la crisis como un desafío que contiene el enorme potencial de lograr el crecimiento personal y familiar.

Palabras clave: Familia, esposos, crisis, separación, divorcio, matrimonio, pareja, conflictos. 
A pesar de las dificultades, las congojas y los desalientos que puedan suscitarse, no abriguen jamás ni el marido ni la mujer el pensamiento de que su unión es un error o una decepción. Elena de White,

\section{Introducción}

Una crisis es considerada como "Percepción de un evento o situación como una dificultad intolerable que excede a los $\mathrm{r}$ ecursos y a los mecanismos de lucha de una persona" (Gilliland y James 1988).

Existen algunas parejas que durante las crisis vit ales o inesperadas olvidan los $\mathrm{v}$ otos matrimoniales yel pact o matrimonial de conf ianza, fidelidad, permanencia y com pañerismo que hicieron en el alt ar. Para prevenir las crisis, o durant e ellas, la par eja debe per severar y luchar constantemente por su vida matrimonial.

\section{Experiencia personal}

Cuando empezamos a criar a nuestra primera niña, enfr entamos una crisis menor que felizmente superamos rápidamente. Es común que cuando un niño se tira al piso, los padr es no saben cómo reaccionar. En nues- tro caso, lo hicimos con conductas diferentes, y esto causó una ligera confusión en la niña. De inmediato hablamos al $r$ especto dejando a un lado nues tras ideas preconcebidas y centrándonos en el bienestar de la niña. Llegamos a un acuer do y empezamos a "hablar el mismo idioma" en relación a la conducta de nuestra pequeña. Esa solución ha funcionado hasta el día de hoy.

En mi experiencia como psicoterapeuta, al analizar casos resueltos y algunos por resolver, he constatado que la mayoría de las parejas experimentan crisis por una inadecuada comunicación, en segundo lugar por casos de infidelidad, y luego por pr oblemas económicos, por celos, insatisfacción sexual, crianza de los hijos y la rutina.

\section{Crisis en el matrimonio}

El matrimonio es un constante aprendizaje. Aun en las parejas mejor consolidadas pueden surgir diferencias en la convivencia, y es que no se trata que nuestra pareja sea apenas la pr olongación de nuestros deseos, sino se trata de complementarse mutuamente, de proveerse el uno 
al otro la felicidad y conser var siempre la dicha de estar juntos, y ese proceso no está libre de dificultades.

La vulnerabilidad o fragilidad frente a las crisis va a depender en gran parte de las caracterís ticas de la personalidad, herencia, experiencias pasadas y car encia de habilidades para $t$ omar decisiones. Estas características frecuentemente debilitan la posibilidad de enfrentar adecuadamente las crisis en cualquiera de sus modalidades.

\section{Las crisis vitales y las crisis situacionales}

Entre los diferentes tipos de crisis sobresalen las llamadas crisis vitales que son aquellas que forman parte del ciclo de vida: la época de $\mathrm{r}$ ecién casados, el inicio de la crianza de los hijos, los desafíos de tener hijos en la etapa preescolar y luego en la etapa escolar, las crisis con hijos adolescentes e hijos jóvenes, las preocupaciones propias de los adultos en la mediana edad y la jubilación. Estas crisis, a pesar que son parte del desarrollo evolutivo del individuo, pueden llegar a producir desde una separación hasta un div orcio legal.
En lo posible, es pr eciso tratar de prevenir o anteponerse a lo que vendrá en cada edad o etapa vivencial.

Por otro lado, es tán también las crisis situacionales o inesperadas como inestabilidad económica, intromisión de los familiares, infidelidad, inestabilidad en la comunicación efectiva y afectiva, separación y divorcio, desgaste en la convivencia, rutina, enfermedad, incompatibilidad de caracteres, muerte, etc.

Cualquiera que sea la situación nunca debemos dejar pasar por alto una crisis por más pequeña que sea, porque con el tiempo ésta irá cr eciendo y se convertirá en una crisis ma yor y será más difícil lidiar con ella. Las crisis menores no resueltas son un peligr o. Pasar por alto estas "pequeñeces" es de alt o riesgo. En el caso de la relación con los hijos, si no se resuelven las crisis a tiem po, puede ser que cuando los padres se den cuenta, el niño ya llegó a la adolescencia, y aquello que antes se hubiera superado con mayor facilidad y en un corto tiempo, se convierte en un descomunal desafío. 
Por otro lado, las peq ueñas crisis no resueltas se van convirtiendo en montañas muy altas, transformándose muchas veces en factores desencadenantes de separación y div orcio. En mi opinión, el divorcio es la última etapa que la pareja enfrenta luego de una serie de situaciones de crisis que no han sido debidamente resueltas. El divorcio, es uno de los golpes finales para la familia.

Cuando la crisis matrimonial golpea, hay ocasiones en que el problema se vuelve tan ingobernable que es difícil saber cómo encararlo y por dónde comenzar. Es evidente que las relaciones en el matrimonio son complejas y que no ha y fórmulas totalmente fijas para usar en este ámbito. Sin duda, en ocasiones será necesario buscar ayuda profesional, pero nunca llegaremos a buen puer to si no em pezamos por lo primer o: reconocer cuál es nuestra parte dentro del problema y qué podemos hacer para resolverlo.

\section{Problemas de comunica- ción en el matrimonio}

La comunicación es un tema al que la pareja siempre le debe prestar atención porque tiene que ver con la expresión de sentimientos y pensamient os. El deseo natural de e xpresar pensamientos y sentimient os, hace necesaria la presencia de alguien muy íntimo como el cónyuge.

La comunicación es la fuente de la intimidad. En la actualidad, los problemas de comunicación se han incrementado por la rutina, el cansancio, el estrés, la ansiedad y un enemigo cruel de la sociedad moderna: la falta de tiempo. En mi e xperiencia profesional he escuc hado muchas veces la frase: "Él jamás me invita a salir a solas". La e xcusa que frecuentemente presentan los esposos es: "no tengo tiempo". Esto ha generado soledad, tristeza y desamparo en la vida de muchas esposas.

Por otro lado, no es raro que, en las parejas que no saben afrontar las crisis, la comunicación esté plagada de palabras destructivas que golpean el alma, así como los golpes letales causan dolor en el cuer po. La comunicación destructiva trae consigo la falta de r espeto hacia la opinión del o tro, el creer que uno siempre tiene la razón y el no poner se en el lugar del 
otro para com prender lo q ue realmente ocurre. Detrás de una palabra destructiva está el veneno del orgullo que va minando la intimidad. Los lazos af ectivos quebrantados y la soberbia de no saber reconocer la falta producen una l ucha constante de ataque y defensa.

La falta de comunicación afectiva se ha convertido en una costumbre para muchas parejas. Hay casos donde la conexión emocional se está perdiendo y apenas existe una comunicación superficial que se limita a la información de gastos, o lo que se comerá al día siguiente, pero se está descuidando la comunicación profunda, el interés por el bienestar emocional del o tro, y el contacto físico y afectivo.

Imagine, apreciado lector, a una pareja sentada frente a una chimenea, recordando momentos lindos y mirando sus $f$ otos; o a una pareja sentada frente al mar prodigándose besos y caricias. Es una imagen linda. Pues bien, es posible que no se cuente con una chimenea, pero hay mil formas en las que una pareja creativa puede disfrutar de una verdadera intimidad. Es necesario huir de la rutina y evit ar las excusas de siempre: "no tengo tiempo", "no me inter esa", etc. Es triste constatar que otras actividades sean las prioridades de una pareja de esposos, mientras su relación conyugal languidece con el cultivo de componentes superficiales que no llenan ni satisfacen.

\section{La infidelidad}

Lamentablemente, en el mundo en que vivimos es muy frecuente la inf idelidad matrimonial. Vivimos en un entorno social de tanta falta de lealt ad que pareciera que ser infiel se ha convertido en algo muy común y cotidiano. La infidelidad altera negativamente toda la dinámica de la familia y se tendrá que recorrer un largo camino para volver a restructurarla.

\section{Crisis relativas al dinero}

La sociedad de consumo promueve una lucha en la que muchas familias están envueltas. Es una lucha por querer tener más, por alcanzar un nivel social más elevado, por "sobresalir" incluso a costa de la propia familia. Esta desgastante lucha materialista es otra carga para la par eja. El mal uso de las tarjetas de crédito, la 
multitud de gastos innecesarios, los inevitables sobregiros mensuales, el pago de las hipo tecas, etc., se convierten en una fuente permanente de reproches, insultos, violencia verbal, agresión física, etc.

\section{La rutina}

Otro de los pr oblemas que afectan más comúnmente al matrimonio es la rutina. Con el paso del tiem po, las par ejas se ven envueltas en la rutina y el aburrimiento. La rutina es uno de los mayores enemigos de cualquier relación. Se hace y se habla siempre lo mismo, se demuestra poco interés en lo que le ocurre al o tro y se cae en la monotonía y la falta de ilusión. Es necesario evitar la pérdida de la alegría y la ilusión por la pareja.

Los esposos deben mantener siempre el deseo de estar juntos y realizar actividades que les interesen. De es ta manera ninguno se sentirá solo o abandonado. Existen matrimonios que, a pesar de comer y dormir juntos, enfrentan una atmósfera de soledad. Es necesario salir de la rutina, ya sea viajando, estableciendo un negocio o cono- ciendo nuevas amistades. Puede ser cualquier actividad, pero lo importante es que la realicen juntos y que ambos disfruten haciéndolo.

También ayuda mucho el tratar de sorprender a la pareja con detalles y nuevas ideas. El amor y la creatividad son los mejor es aliados para vencer a la rutina.

\section{Elementos de prevención y confrontación frente a la crisis}

Los problemas conyugales son muchos y tendríamos que tratarlos por separado. Sin embargo, me gus taría detallar algunas recomendaciones que servirán como elementos de prevención y ayuda al momento de enfrentar las crisis.

En primer lugar se tiene q ue identificar y reconocer la crisis. No sirve de nada tratar de guardar las apariencias viviendo la fantasía del "matrimonio f eliz" cuando lo que existe realmente es un grito desesperado por ayuda. Además, es necesario saber diferenciar si se trata de una crisis vital o una situacional. En el caso de las crisis vitales, es necesario anticiparse a tra vés de 
una preparación permanente, y mantener una visión realista del periodo o etapa de vida que les corresponderá vivir en un cor to o mediano plazo. Es ta preparación requiere de hacer planes para el futuro tanto a nivel personal como a nivel de pareja.

En el caso del surgimiento de crisis con los hijos en las diferentes etapas de su cr ecimiento, es recomendable buscar fuentes de información confiables tanto científica como espiritualmente. También ayudaría mucho buscar consejería y orientación con profesionales del área.

Un segundo aspecto es la unidad. La par eja debe es tar más unida que nunca, pr ocurando pasar juntos el mayor tiempo posible, compartiendo salidas y pasatiempos. Es necesario mantener una comunicación abierta y sincera, y entender que el amor que puso Dios en sus corazones es más fuerte que cualquier crisis. También ayudará mucho el mantener la calma y el buen humor, y el pr ocurar escuchar a personas que ya ha $\mathrm{n}$ experimentado alguna crisis vit al. En esto último, la par eja puede escuchar, analizar y rescatar lo que sirva para el fortalecimiento de su relación. También ayuda mu- cho el aprender a tener tolerancia, ya que la tolerancia mutua capacita a los cónyuges para vivir juntos en paz, armonía y amor.

En el caso de las crisis inesperadas sería bueno considerar lo siguiente: recordar el compromiso asumido en los $\mathrm{v}$ otos matrimoniales. Además, es bueno que cada cónyuge se esmere para que su casa sea un verdadero hogar, dando lugar a las palabras de bondad, cordialidad, y respeto. También deberían cultivar el interés del uno por el o tro, encontrando algunos pasatiem pos como leer algún libro, salir a caminar o a pasear. Haga que cada día el amor sea creativo.

Por otro lado, es recomendable marcar en su agenda familiar las horas de pláticas y, dentro de ellas, los momentos de oración y lectura de text os edificantes que les ayudarán a fortalecer sus emociones. Los esposos deben cuidar que, por más fuerte sea la crisis, su fe en Dios nunca decaiga. Los esposos deben pr ocurar ser solucionadores de pr oblemas, identificando con claridad las dificultades y evitando buscar culpables. Además, es necesario centrarse en la solución teniendo los roles bien definidos 
y siendo flexibles en caso de ser necesario.

En los casos de separación, divorcio, infidelidad u otra crisis de tipo mayor lo mejor es buscar ayuda terapéutica y espiritual.

Es necesario $r$ emarcar que el problema no está en la crisis propiamente dicha, sino en la percepción que se tiene de ella y en los recursos que utiliza la pareja para confr ontar o afr ontar dichas crisis. Cuando la par eja ve a esa crisis como una opor tunidad de crecimiento personal y familiar, su visión será op timista; pero si la per cibe como una desgracia se generará una visión catastrófica. Cuando se llega a una situación en la q ue se trascienden los recursos usados por el individuo, ésta será realmente una crisis.

Filipenses 4:1 3 nos dice: "Todo lo puedo en Cristo que me fortalece", esto indica que es posible tener el contr ol de la situación aun en medio de una crisis. Un matrimonio no tiene por qué estar condenado al fracaso, al contrario es posible alcanzar las cumbres de un amor profundo, verdadero y abnegado.

\section{Conclusión}

Para alcanzar el obje tivo de tener un matrimonio $f$ eliz, es necesaria una vida consagrada y dedicada a Dios. Él debe ocupar el primer lugar en nues tra vida. Ser r ealmente el primero en nuestros afectos. Cuando el amor de Dios mora en el corazón de cada esposo y esposa, ellos estarán en condición de enfrentar victoriosamente las crisis de su vida matrimonial. La presencia de Cristo en el hogar convertirá a los padres y cónyuges en per sonas íntegras y con autoridad frente a sus hijos y la sociedad. Entonces es tiempo de colocar a Cristo en el tr ono de nuestro hogar. 